\title{
Overview of the COST Action TD1105 EuNetAir
}

\author{
Michele Penza \\ ENEA - Italian National Agency for New Technologies, Energy and Sustainable Economic \\ Development - Technical Unit Brindisi Technologies for Materials, PO Box 51 Br4, 72100 Brindisi, Italy \\ Corresponding author: michele.penza@enea.it
}

\begin{abstract}
This is a short overview of the COST Action TD1105 EuNetAir - European Network on New Sensing Technologies for Air-Pollution Control and Environmental Sustainability - funded in the framework European Cooperation in the field of Scientific and Technical Research (COST) during the period 2012-2016. The main objective of the Concerted Action is to develop new sensing technologies for Air Quality Control at integrated and multidisciplinary scale by coordinated research on nanomaterials, sensor-systems, air-quality modelling and standardised methods for supporting environmental sustainability with a special focus on Small and Medium Enterprises. This international Networking, coordinated by ENEA (Italy), includes over 60 big institutions from 20 COST Countries (EU-zone) and 5 Non-COST Countries (extra-Europe) to create a S\&T critical mass in the environmental issues.
\end{abstract}

Key words: Functional materials, sensor nanomaterials, gas sensors, smart sensor systems, environmental measurements/modeling, protocols and standards, air quality control.

\section{Introduction}

This COST Action [1] focuses on a new detection paradigm based on sensing technologies at low cost for Air Quality Control (AQC) and set up an interdisciplinary top-level coordinated network to define innovative approaches in sensor nanomaterials, gas sensors, wireless sensor-systems, distributed computing, methods, models, standards and protocols for environmental sustainability within the European Research Area (ERA).

The state-of-the-art research on innovative sensing technologies for AQC based on advanced chemical sensors and sensorsystems at low-cost, including functional materials and nanotechnologies for ecosustainability applications, the outdoor/indoor environment control, olfactometry, air-quality modeling, chemical weather forecasting, and related standardisation methods is performed already at the international level, but still needs serious coordination efforts to boost new sensing paradigms for research and innovation. Only a close multidisciplinary cooperation will ensure cleaner air in Europe and reduced negative effects on human health for the future generations in the smart cities, efficient management of the green buildings at low $\mathrm{CO}_{2}$ emissions, including sustainable economic development.

The objective of the Action (see logo in Fig. 1) is to create a cooperative network to explore new sensing technologies for low-cost airpollution control through field studies and laboratory experiments, to transfer the results into preventive real-time control practices and to move towards global sustainability via climate change monitoring and outdoor/indoor energy efficiency. Establishment of such a network, involving COST Countries participants as well as Non-COST key-experts, will enable Europe to develop world capabilities in urban sensor technology based on cost-effective nanomaterials, to form a critical mass of researchers suitable for cooperation in science and technology, to give training and education, to coordinate outstanding $R \& D$, to promote innovation towards industry, and to support policy-makers and regional decision-makers.

European Network on New Sensing Technologies for AirPollution Control and Environmental Sustainability - EuNetAir

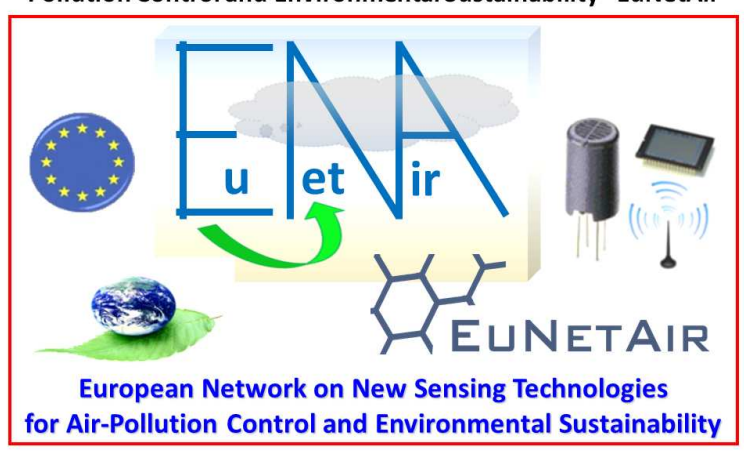

Fig. 1. COST Action TD1105 EuNetAir Logo. 


\section{Objectives}

The aim of the Action is to form a Europeanwide science and technology knowledge platform by a multidisciplinary coordinated network at international level on the new sensing technologies for Air Quality Control including sensor nanomaterials, portable wireless sensor-systems and distributed computing, air-quality modeling and chemical weather forecasting, standards, methods and protocols for environmental measurements in order to advance R\&D and innovation in the European green-economy by strengthening the sustainable development in smart cities, outdoor air-pollution control and indoor energy efficiency in buildings and to foster the technology transfer of the new sensing paradigm of the cost-effective chemical sensors in the European Countries with a special focus on SMEs. With the participation of researchers and industry, as primary objective, the Action will develop a transnational network of multidisciplinary expertise in AQC from COST and Non-COST Countries, to investigate novel sensors and advanced deployment technologies for environmental monitoring.

The main objectives of the Action are listed, but not limited to:

- to establish a top-level Pan-European multidisciplinary $R \& D$ platform on new sensing paradigm for AQC contributing to sustainable development, green-economy and social welfare

- to create collaborative research teams in the ERA on the new sensing technologies for $A Q C$ in an integrated approach to avoid fragmentation of the research efforts

- to train early stage researchers (ESR) and new young scientists in the field for supporting competitiveness of European industry by qualified human potential

- to promote gender balance and involvement of ESR in AQC

- to disseminate R\&D results on AQC towards industry community and policy makers as well as general public and high schools.

The secondary objectives, across the four Working Groups of the Action, are:

- to provide a platform between scientists in the field of materials, nanotechnology and sensor-systems and other scientists such as environmental protection engineers, public agencies managers, stakeholders, decisionmakers, aiming to improve best practices in $A Q C$ and explore the potential role of new generation of low-cost sensing devices
- to investigate sensing mechanisms of the functional nanomaterials for the gas measurement and identification of the best available nanomaterials, proving concepts and harmonizing pre-standardised methods; based on available datasets from partners

- to assess degradation rates and lifetime of sensor elements in defined environmental conditions and evaluate interactions of sensitive materials with outdoor/indoor pollutants; based on datasets from ongoing and historical field deployments of low cost sensors

- to investigate the best available technology for sensor deployment, communication, power supply and data storage, analysis and display

- to monitor real-world environmental conditions with experimental campaigns to assess composition of indoor air (buildings: house and office) and outdoor air (urban areas and industrial sites) and to investigate how such data can be utilised in air pollution modeling

- to approach standardisation of methods for air quality measurements, e.g. harmonization of test procedures, chemical analyses, post-processing, protocols etc.

- to disseminate knowledge on functional materials and sensor-systems for AQC; to aid better focusing of Europe's resources by coordinated efforts in the $A Q C$ and environmental sustainability to strengthen Europe's competitiveness and scientific excellence improving capacity building and networking to tackle global challenges in a big market in the mid-long term.

Selected research products and innovative technologies developed by the partnership of COST Action TD1105 are shown in the Fig. 2.

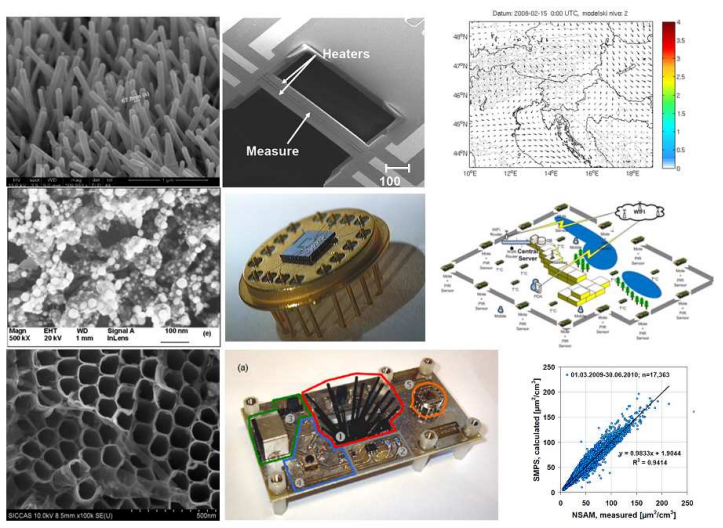

Fig. 2. Selected R\&D technological products by some partners involved in the COST Action TD1105 EuNetAir. Courtesy from EuNetAir Partnership. 


\section{Scientific Programme}

This COST Action focuses on the new sensing technologies for low-cost AQC. This is of strategic importance for the development of European industrial capability in environmental sustainability and green-economy, and to protect the public health. In the past, noticeable scientific efforts have been realized to develop functional materials and devices for AQC, airpollution modeling and chemical forecasting, but developed nanosensors for cost-effective AQC have not yet commercialized.

The national research projects in this field have to be coordinated in this Action aiming to share roadmaps and common purposes. In fact, the national efforts, coherently with the research projects worldwide, are considered more promising for the technological applications in a global scenario at an international level.

The Action will coordinate different research tasks to achieve the objectives described in previous section. The Workplan is organized in four complementary Working Groups (WGs), each devoted to a progressive development of synthesis, characterization, fabrication, integration, prototyping, proof-of-concepts, modeling, measurements, methods, standards, tests and application aspects. The four WGs with the specific objectives are:

- WG1: Sensor materials and nanotechnology

- WG2: Sensors, devices and sensor-systems for AQC

- WG3: Environmental measurements and airpollution modeling

- WG4: Protocols and standardisation methods

Initially, this Action will focus on the study of sensor nanomaterials and nanotechnologies exhibiting unique properties in terms of chemical and thermal stability, high sensitivity, selectivity. Nanosize effects of functional materials will be explored for integration in the gas sensors at low power-consumption. Furthermore, specific nanostructures with tailored sensing properties will be developed for gas sensors and sensor-systems with advanced functionalities.

Enhanced microsensors and nanosensors with functional nanomaterials will be studied in sensor networks at distributed deployment with high spatial and temporal resolution and wireless communications of data. Improved airpollution models based on real-time datasets from deployed sensor networks will provide high-resolution mapping of air-pollutants to provide accurate chemical weather forecasting and air-pollution modeling. Long-term experimental campaigns of portable sensor systems by environmental measurements in field are expected to assess the huge potential of the new sensing technologies for costeffective AQC.

\section{Working Groups}

Generally, each Working Group (WG) will have a Committee lead by a Chair with a Vice-Chair including Sub-Working Group Leaders (SWGL). The Sub-Working Groups (SWG) in each WG are defined as follows:

- WG1: Sensor materials and nanotechnology

1.1 SWG 1.1: Metal oxides nanostructures for $A Q C$ gas sensors

1.2 SWG 1.2: Carbon nanomaterials for $A Q C$ gas sensors

1.3 SWG 1.3: Emerging sensor materials (organic/inorganic, functional, hybrid, nanocomposites, polymers)

- WG2: Sensors, devices \& systems for AQC

2.1 SWG 2.1: Gas nanosensors and new transducers

2.2 SWG 2.2: Portable gas sensor-systems

2.3 SWG 2.3: Wireless technology and AQC sensors network

2.4 SWG 2.4: Intelligence algorithms and distributed computing for networked AQC gas sensors

- WG3: Environmental measurements and airpollution modeling

3.1 SWG 3.1: Environmental measurements at laboratory and in field air-quality stations

3.2 SWG 3.2: Air-quality modeling and chemical weather forecasting

3.3 SWG 3.3: Harmonisation of environmental measurements

- WG4: Protocols \& standardisation methods

4.1 SWG 4.1: Protocols, standards and methods for AQC by analyzers and instruments (no-sensors) technologies

4.2 SWG 4.2: Protocols, standards and methods for AQC by sensors (noanalyzers) technologies

4.3 SWG 4.3: Benchmarking of new products and market of commercial $A Q C$ sensors.

In addition to the mentioned four WGs, several Special Interests Groups (SIGs) devoted to specific issues with interdisciplinary contents will be setup to pursue planned objectives involving research, academia and industry people regarding gender balance and including early stage researchers in the Action agenda. A flow-chart of the COST Action TD1105 structure and organization is depicted in the Fig. 3. 


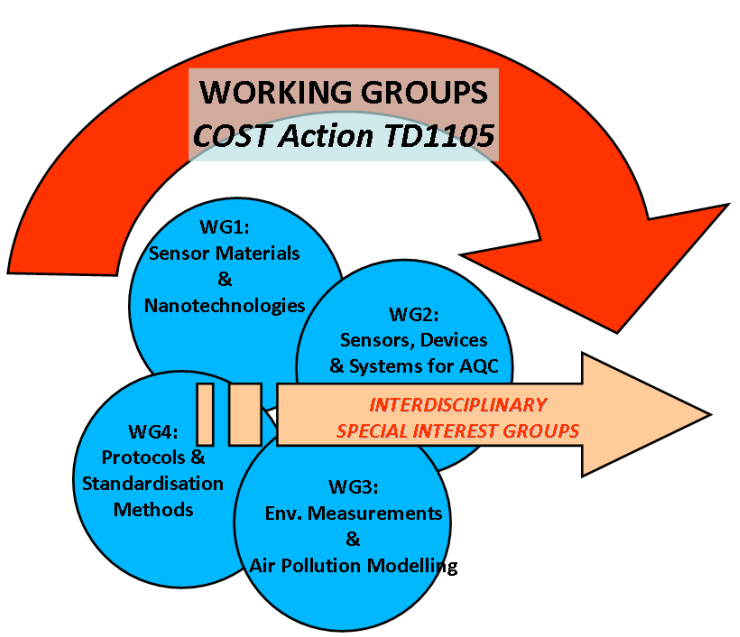

Fig. 3. Working Groups (WGs) and Special interest Groups (SIGs) in COST Action TD1105 EuNetAir.

\section{Participants of COST Action TD1105}

At the moment of approval of the Action, 51 big Institutions from 17 European Countries (Belgium, Bulgaria, Switzerland, Germany, Denmark, Greece, Spain, Finland, France, Hungary, Italy, Lithuania, Netherlands, Poland, Sweden, Slovenia, United Kingdom) participated in the preparation of the proposal. The Action spans largely across the European Union including a wide geographical coverage and other Countries, such as Norway, Iceland, Latvia, Romania, signed MoU after its approval from CSO. At the date on April 2012, 20 European Countries were participants in the COST Action TD1105 by involving 60 research teams from COST area (Europe-zone). The Action participants are from 30 Universities, 13 Research Centers, 2 Environmental Agencies and 15 SMEs including 5 spin-offs.

Additional 6 top-level Institutions from 5 NonCOST Countries (Australia, USA, Canada, China, Russia) were involved to Action: CSIRO, Chinese Academy of Sciences, National Research Center Kurchatov-Institute, University of Waterloo, Southern Illinois University Carbondale, NASA Ames Nano Research Center.

Participating Institutions are: Universitè de Liège (BE); VITO (BE); Odometric SA (BE); Bulgarian Academy of Sciences (BG); Ecole Polytechnique Federale de Lausanne $(\mathrm{CH})$; E2V Microsensors SA (CH); EnvEve SA (CH); Empa Swiss Federal Laboratories for Materials Science and Technology $(\mathrm{CH})$; Institute of Energy and Environmental Technology (DE); Alfred Becker $\mathrm{GmbH}$ (DE); $3 \mathrm{~S} \mathrm{GmbH}$ (DE); Saarland University (DE); University of Bayreuth (DE); University of Paderborn (DE); UST GmbH (DE); Aarhus University (DK); Technical University of Denmark (DK); Aristotle
University (EL); FORTH (EL); ISI-ATHENA (EL); Catalonia Institute for Energy Research (ES); CSIC (ES); Universitat Rovira i Virgili (ES); University of Barcelona (ES); Worldsensing SL (ES); University of Oulu (FI); University of Helsinki (FI); University of Tampere (FI); Universitè de Bourgogne (FR); University Blaise Pascal (FR); Hungarian Meteorological Service (HU); Agricultural University of Iceland (IS); ENEA (IT); ELETTRA (IT); University of Bari (IT); Lenviros srl (IT); Sensichips srl (IT); University of Brescia (IT); University of Trieste (IT); University of Latvia (LV); Lithuania Environmental Protection Agency (LT); IMEC (NL); Norwegian Institute for Air Research (NO); Silesian University of Technology (PL); Warsaw University of Life Science (PL); National R\&D Institute for Nonferrous and Rare (RO); SC IPA SA (RO); Metals Chalmers University of Technology (SE); Linkoping University (SE); SenseAir AB (SE); SenSiC AB (SE); Aerosol doo (SI); University of Ljubljana (SI); Alphasense Ltd (UK); Cambridge CMOS Sensors Ltd (UK); Imperial College London (UK); Newcastle University (UK); University of Manchester (UK); University of Warwick (UK); University of Cambridge (UK).

\section{Conclusions}

This COST Action TD1105 seems to be the best approach to coordinate, streamline, integrate and harmonize the interaction between material scientists, environmental modellers, chemists, computing engineers, sensor manufacturers, end-users, stakeholders for a wide international community to address environmental issues and to establish European leadership on Air Quality Control technologies to support green-economy and competitiveness of the European industry.

\section{Acknowledgements}

This COST Action TD1105 EuNetAir has been approved by the COST Committee of Senior Officials (CSO) at its $183^{\text {rd }}$ meeting on 1 December 2011 at Brussels. Memorandum of Understanding (MoU) oc-2011-1-9706 has been signed by 20 COST Countries Governments at the date on April 2012 in the COST Domain Earth System Science and Environmental Management (ESSEM) - TransDomain Action. The proposer/chair is highly indebted to All Partners for excellent scientific interests and expertise to support the Action.

\section{References}

[1] Action Fact Sheet: http://www.cost.eu/domains_actions/essem/Actio ns/TD1105. 